\title{
Clinical outcomes after sentinel lymph node biopsy in clinically node-negative breast cancer patients
}

\author{
Hee Ji Han, MD', Ju Ree Kim, MD, PhD², Hee Rim Nam, MD, PhD³, \\ Ki Chang Keum, MD, PhD', Chang Ok Suh, MD, PhD', Yong Bae Kim, MD, PhD \\ 'Department of Radiation Oncology, Yonsei Cancer Center, Yonsei University College of Medicine, Seoul; \\ ${ }^{2}$ Department of Radiation Oncology, Cheil General Hospital, Seoul; ${ }^{3}$ Department of Radiation Oncology, \\ Kangbuk Samsung Hospital, Sungkyunkwan University School of Medicine, Seoul, Korea
}

Purpose: To evaluate non-sentinel lymph node (LN) status after sentinel lymph node biopsy (SNB) in patients with breast cancer and to identify the predictive factors for disease failure.

Materials and Methods: From January 2006 to December 2007, axillary lymph node (ALN) dissection after SNB was performed for patients with primary invasive breast cancer who had no clinical evidence of LN metastasis. A total of 320 patients were treated with breast-conserving surgery and radiotherapy.

Results: The median age of patients was 48 years, and the median follow-up time was 72.8 months. Close resection margin (RM) was observed in 13 patients. The median number of dissected SNB was two, and that of total retrieved ALNs was 11. Sentinel node accuracy was $94.7 \%$, and the overall false negative rate (FNR) was 5.3\%. Eleven patients experienced treatment failure. Local recurrence, regional LN recurrence, and distant metastasis were identified in $0.9 \%, 1.9 \%$, and $2.8 \%$ of these patients, respectively. Sentinel LN status were not associated with locoregional recurrence $(p>0.05)$. Close RM was the only significant factor for disease-free survival (DFS) in univariate and multivariate analysis. The 5 -year overall survival, DFS, and locoregional DFS were 100\%, $96.8 \%$, and $98.1 \%$, respectively.

Conclusion: In this study, SNB was performed with high accuracy and low FNR and high locoregional control was achieved.

Keywords: Breast neoplasms, Sentinel lymph node biopsy, Axillary lymph node dissection, Neoplasm recurrence, Local

\section{Introduction}

In invasive breast cancer, axillary lymph nodal status is considered to be an important prognostic factor for predicting clinical outcome. Since the 1990s, sentinel lymph node biopsy (SNB) has been performed routinely for evaluating axillary lymph node (ALN) status and reducing complications of axillary lymph node dissection (ALND). In several studies,
SNB could accurately predict ALN status in clinically nodenegative breast cancer patients. Only 5\%-15\% of early breast cancer patients with negative sentinel lymph node (SLN) had additional ALN metastasis [1-3]. Because ALND increases the risk of complications, such as lymphedema, chronic shoulder pain, arm weakness and joint dysfunction without survival benefit, completion ALND is not routinely performed in SLNnegative patients $[2,4,5]$.

Received 21 April 2014, Revised 17 June 2014, Accepted 1 July 2014.

Correspondence: Yong Bae Kim, MD, PhD, Department of Radiation Oncology, Yonsei Cancer Center, Yonsei University College of Medicine, 50 Yonsei-ro, Seodaemun-gu, Seoul 120-749, Korea. Tel: +82-2-2228-8095, Fax: +82-2-2227-7823, E-mail:YBKIM3@yuhs.ac

(c) This is an Open Access article distributed under the terms of the Creative Commons Attribution Non-Commercial License (http://creativecommons.org/ licenses/by-nc/3.0/) which permits unrestricted non-commercial use, distribution, and reproduction in any medium, provided the original work is properly cited.

www.e-roj.org 
In patients with positive SLN, additional ALN involvement is observed in 40\%-60\%. Because additional ALN metastasis is considered to be a significant risk factor for disease progression, completion ALND is recommended for regional control in SLN-positive patients [6]. However, several prospective trials have shown that the regional failure rate is lower than expected in patients without ALND who received SNB only based on the incidence of axillary metastases. No survival difference was observed between patients with SNB only and patients with SNB and completion ALND or regional radiotherapy [7-10]. Based on the result of these clinical trials, additional ALND may be minimized in clinically node-negative patients with T1 and T2. The purpose of this study was to investigate the association between SLN status and ALN status in clinically node-negative early breast cancer patients and to evaluate the clinical outcomes after SNB and ALND in our institution.

\section{Materials and Methods}

We retrospectively performed a chart review of primary breast cancer patients treated with whole breast radiotherapy at our hospital, between January 2006 and December 2007. The criteria for entry into this study were as follows: 1) pathologically proven invasive breast cancer, 2) no clinical evidence of lymph node metastasis, 3) performance of breastconserving surgery, SNB, and ALND, and 4) completion of whole breast radiotherapy with or without regional node irradiation. We excluded patients who were male; had a history of breast irradiation, stage IV or recurrent breast cancer and who underwent neoadjuvant chemotherapy. A total of 320 patients were eligible.

For staging work-up, all patients received mammography, ultrasound of breast, abdomen and whole body bone scan. In some patients, breast magnetic resonance imaging was done for work-up. We defined clinically node-negative (cNo) as a no evidence of lymph node metastasis in the preoperative imaging study and physical examination. Two patients with palpable ALN or typical metastatic lymph node (round shape, obliterated hilum) received fine needle aspiration biopsy, and the result was negative. SLN detection was only performed using the radio-isotope technique. On the day of surgery, 18.5 $\mathrm{MBq}$ (0.5 mCi) 99mTc-Phytate (Korea Atomic Energy Research Institute, Daejeon, Korea) diluted in $0.5 \mathrm{~mL}$ saline was injected at the upper outer subareolar. A Iymphatic scintigram was obtained for each patient. In the operating room, a hand-held gamma detector (Navigator GPS; RMD Inc., Watertown, MA, USA) was used to locate the SLNs. Four patients who fail SLN detection were excluded from this study.

ALND was performed for all patients following SNB. During this period, completion ALND performed even if the SLN was negative, because surgeons wanted to make sure negative ALN after negative SLN. All enrolled patients were staged according to the American Joint Committee on Cancer (AJCC) 7th staging system. A surgical margin was diagnosed as close if tumor were $2 \mathrm{~mm}$ or less from postsurgically applied inked margin.

All analyses were performed using SPSS software ver. 20.0

Table 1. Patient characteristics

\begin{tabular}{|c|c|c|}
\hline \multicolumn{2}{|c|}{ Characteristic } & No. of patients (\%) \\
\hline \multicolumn{2}{|c|}{ Age (yr), median (range) } & $48(25-76)$ \\
\hline \multicolumn{3}{|l|}{ AJCC stage } \\
\hline IA & T1N0M0 & $189(59.1)$ \\
\hline IB & T1N1miM0 & $13(4.1)$ \\
\hline \multirow[t]{2}{*}{$\| A$} & T1N1M0 & $43(13.4)$ \\
\hline & T2N0MO & $43(13.4)$ \\
\hline$\| B$ & T2N1M0 & $21(6.6)$ \\
\hline \multirow[t]{3}{*}{$\| I A$} & T1N2M0 & $5(1.6)$ \\
\hline & T2N2Mo & $5(1.5)$ \\
\hline & T3N1M0 & $1(0.3)$ \\
\hline \multicolumn{3}{|c|}{ Estrogen receptor status } \\
\hline \multicolumn{2}{|l|}{ Positive } & $220(68.8)$ \\
\hline \multicolumn{2}{|l|}{ Negative } & 99 (30.9) \\
\hline \multicolumn{2}{|l|}{ Unknown } & $1(0.3)$ \\
\hline \multicolumn{3}{|c|}{ Progesterone receptor status } \\
\hline \multicolumn{2}{|l|}{ Positive } & $199(62.2)$ \\
\hline \multicolumn{2}{|l|}{ Negative } & $120(37.5)$ \\
\hline \multicolumn{2}{|l|}{ Unknown } & $1(0.3)$ \\
\hline \multicolumn{3}{|l|}{ HER2 } \\
\hline \multicolumn{2}{|l|}{$0,1+, 2+$} & $280(87.5)$ \\
\hline \multicolumn{2}{|l|}{$3+$} & $39(12.2)$ \\
\hline \multicolumn{2}{|l|}{ Unknown } & $1(0.3)$ \\
\hline \multicolumn{3}{|l|}{ Histology } \\
\hline \multicolumn{2}{|l|}{ IDC } & $288(90.0)$ \\
\hline \multicolumn{2}{|l|}{ Other } & $32(10.0)$ \\
\hline \multicolumn{3}{|c|}{ Extensive intraductal component } \\
\hline \multicolumn{2}{|l|}{ No } & $246(76.9)$ \\
\hline \multicolumn{2}{|l|}{ Yes } & $51(15.9)$ \\
\hline \multicolumn{2}{|l|}{ Unknown } & $23(7.2)$ \\
\hline \multicolumn{3}{|c|}{ Histologic grade } \\
\hline \multicolumn{2}{|c|}{1} & $71(22.2)$ \\
\hline \multicolumn{2}{|l|}{2} & $143(44.7)$ \\
\hline \multicolumn{2}{|l|}{3} & $75(23.4)$ \\
\hline \multicolumn{2}{|l|}{ Unknown } & $31(9.7)$ \\
\hline
\end{tabular}

AJCC, American Joint Committee on Cancer; HER2, human epidermal growth factor receptor 2; IDC, invasive ductal carcinoma. 
(IBM, Armonk, NY, USA). Overall survival (OS), disease-free survival (DFS), locoregional DFS (LRDFS) were analyzed using the Kaplan-Meyer method and univariate analysis of clinical factors influencing each failure were analyzed using the logrank test. Multivariate analysis was performed using the Coxproportional hazard model. The $p$-values less than 0.05 were considered statistically significant.

\section{Results}

\section{Patient characteristics}

Patient characteristics are shown in Table 1. The median age was 48 years (range, 25 to 76 years). According to AJCC

Table 2. Treatment characteristics

\begin{tabular}{lc}
\hline \multicolumn{1}{c}{ Characteristic } & No. of patients (\%) \\
\hline No. of dissected SLNs & $2(1-16)$ \\
1 & $133(41.6)$ \\
2 & $85(26.6)$ \\
3 & $42(13.1)$ \\
4 & $30(9.4)$ \\
5 & $14(4.4)$ \\
$\geq 6$ & $16(5.0)$ \\
No. of metastatic SLNs & \\
0 & $251(78.4)$ \\
1 & $51(15.9)$ \\
2 & $11(3.4)$ \\
3 & $3(0.9)$ \\
$\geq 4$ & $4(1.3)$ \\
No. of dissected ALNs & $11(1-35)$ \\
Additional involved nodes & \\
No & $282(88.1)$ \\
Yes & $38(11.9)$ \\
Close or positive RM & \\
No & $307(95.9)$ \\
Yes & $13(4.1)$ \\
\hline
\end{tabular}

Values are presented as median (range) or number (\%). SLN, sentinel lymph node; ALN, axillary lymph node; RM, resection margin. 7th stage, the number of patients with stage $\mid A, I B, \| A$, II , and IIIA was 189 (59.1\%), 13 (4.1\%), 86 (26.9\%), 21 (6.6\%), and $11(3.4 \%)$, respectively. The most common histologic type of tumor was invasive ductal carcinoma $(288,90.0 \%)$. Estrogen receptor positive, progesterone receptor positive, and c-erbB2 3+ was found in 220 patients (68.8\%), 199 (62.2\%), and $39(12.2 \%)$, respectively. In c-erbB2 $2+$ patients by immunohistochemistry, FISH or SISH was done for 7 patients (26\%). Among them, c-erbB2 amplification by FISH or SISH was observed in one patient. Extensive intraductal component was found in 51 patients (15.9\%).

\section{Treatment characteristics}

Treatment characteristics are summarized in Table 2. The median number of removed nodes was two (range, 1 to 16), and positive SLN was found in 69 patients (21.6\%). The median number of removed ALNs was 11 (range, 1 to 35), and additional involved lymph nodes were found in 38 patients (11.9\%). Among the patients with negative SLN, positive conversion in permanent section was shown in two patients. Close or positive resection margin (RM) were found in 13 patients (4.1\%). All patients received whole breast irradiation with median of $50.4 \mathrm{~Gy}$ in 28 fractions, and 313 patients $(97.8 \%)$ received a tumor bed boost with a median dose of 9 Gy. Treatment included the breast only in 294 patients (91.9\%), breast and supraclavicular lymph node (SCL) in 26 patients with positive axillary node (8.1\%), and breast, SCL, and internal mammary lymph node in 20 patients with inner/central tumor with or without positive axillary node (6.3\%).

Most patients $(300,93.6 \%)$ received systemic treatments; chemotherapy alone in 83 patients (25.9\%), hormonal therapy alone in 81 patients (25.3\%), and both in 133 patients (41.6\%). Among the 219 patients who received chemotherapy, doxorubicin and cyclophosphamide (AC) were used in 124 patients (56.6\%); doxorubicin, cyclophosphamide, and paclitaxel (ACT) in 64 patients (29.2\%), and cyclophosphamide, methotrexate, and 5-fluorouracil (CMF) in 17 patients (7.8\%).

Table 3. FNR rate according to the number of dissected SLNS

\begin{tabular}{ccccccc}
\hline No. of dissected SLNs & No. of patients & TN & FN & TP & FNR (\%) & Accuracy (\%) \\
\hline 1 or more & 320 & 234 & 17 & 21 & 5.3 & 94.7 \\
2 or more & 187 & 136 & 9 & 12 & 9 & 1.3 \\
3 or more & 102 & 70 & 3 & 7 & 0 & 96.1 \\
4 or more & 60 & 40 & 0 & 100 \\
\hline
\end{tabular}

SLN, sentinel lymph node; TN, true negative; FN, false negative; TP, true positive; FNR, false negative rate. 
Two hundred fourteen patients who received hormonal therapy; tamoxifen in 157 patients (73.4\%) and letrozole in 45 (21.0\%).

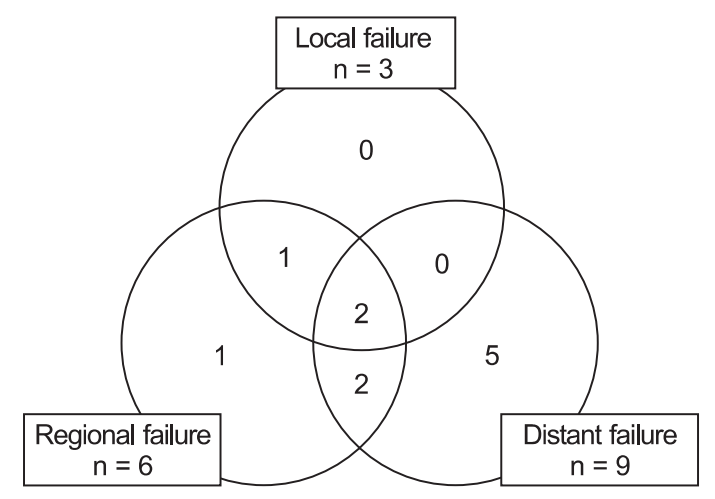

Fig. 1. Patterns of failure. Eleven patients developed treatment failure; local recurrence, regional lymph node recurrence, and distant metastasis were identified in three patients (0.9\%), six (1.9\%), and nine (2.8\%), respectively.

A

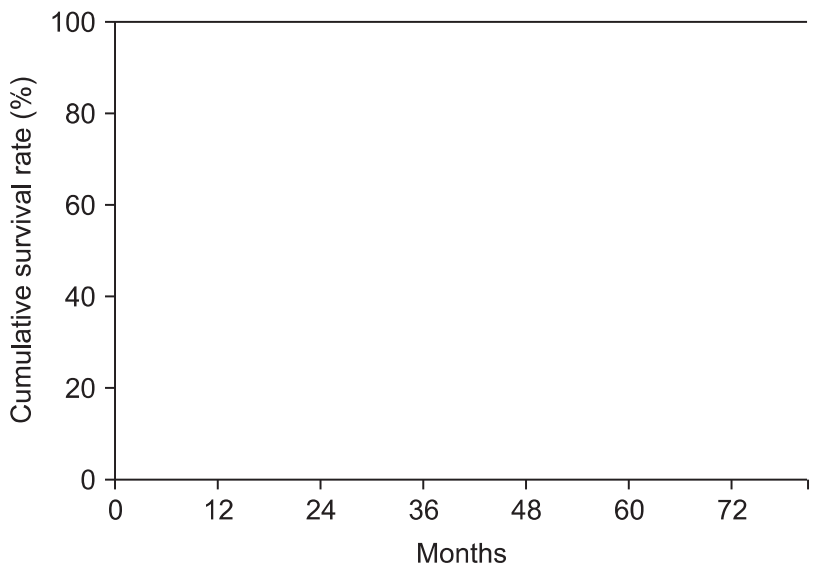

C

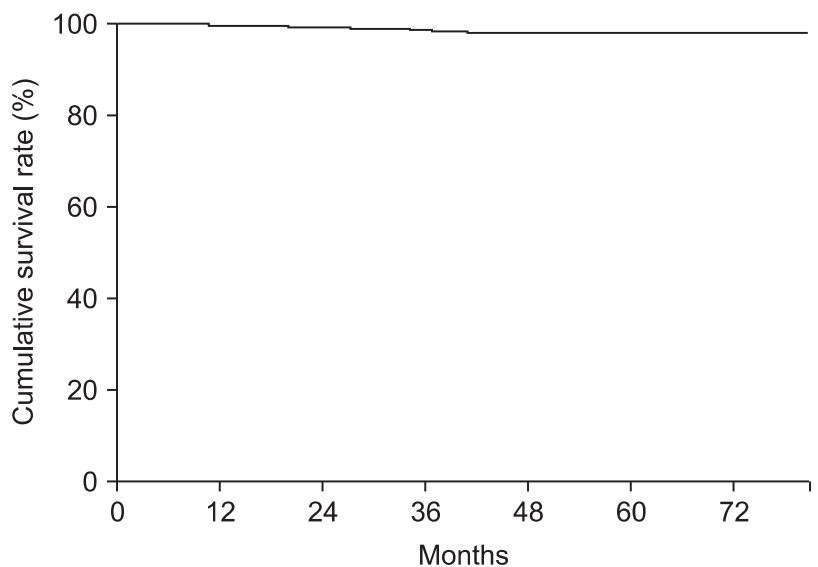

3. Accuracy and false negative rate of sentinel node biopsy SNB and ALND were performed in all patients. Sixty-nine patients had positive sentinel node, and additional involved nodes were shown in 38 patients. Table 3 shows the accuracy and false negative rate (FNR) according to the number of dissected SLNs. The sentinel node accuracy was 94.7\% and the FNR was 5.3\% in all patients with ALND. The FNR of the biopsy decreased as the number of removed lymph nodes increased. Specifically, the FNR was $0 \%$ when more than four lymph nodes were removed. There were 21 true positive, 234 true negative, and 17 false negative results.

\section{Survival outcomes, patterns of failure, and prognostic factors}

With a median follow-up of 72.8 months (range, 6 to 91 months), 11 patients (3.4\%) experienced disease progression during the follow-up period. The patterns of failure are illustrated in Fig. 1. Local recurrence, regional lymph node recurrence and distant metastasis were identified in three

\section{B}

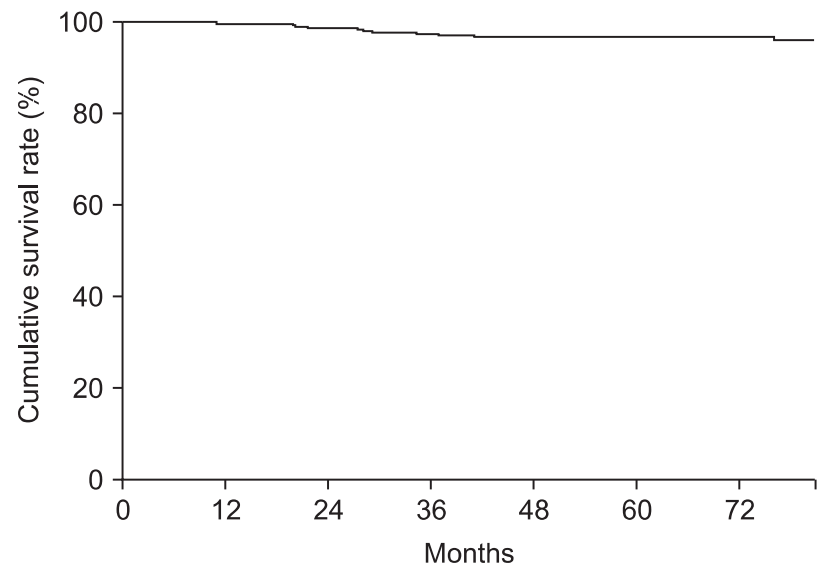

Fig. 2. (A) Overall survival (OS), (B) disease-free survival (DFS), and $(C)$ locoregional DFS among all patients. The 5-year OS, DFS, and locoregional DFS were 100\%, 96.8\%, and 98.1\%, respectively. 
patients $(0.9 \%)$, six $(1.9 \%)$ and nine $(2.8 \%)$, respectively. Locoregional recurrence occurred in six patients. Among them, only two patients had locoregional recurrence without distant metastasis. The 5-year OS, DFS, and LRDFS were 100\%, 96.8\%, and $98.1 \%$, respectively (Fig. 2). In univariate and multivariate analyses, close RM was the only significant factor for lower DFS ( $p=0.014)$ (Table 4). Neither SLN nor non-SLN status was significantly associated with locoregional recurrence and disease recurrence $(p>0.05)$.

Table 4. Univariate and multivariate associations of prognostic factors with recurrence-free survival

\begin{tabular}{|c|c|c|c|}
\hline \multirow[b]{2}{*}{ Variables } & \multirow{2}{*}{$\begin{array}{c}\text { No. of } \\
\text { patients }\end{array}$} & \multicolumn{2}{|c|}{ p-value } \\
\hline & & Univariate & $\begin{array}{l}\text { Multi- } \\
\text { variate }\end{array}$ \\
\hline Age (yr) & & 0.463 & 0.221 \\
\hline$<50$ & 199 & & \\
\hline$>50$ & 121 & & \\
\hline T stage & & 0.879 & 0.648 \\
\hline $\mathrm{T} 1$ & 250 & & \\
\hline $\mathrm{T} 2$ & 69 & & \\
\hline T3 & 1 & & \\
\hline SLN status & & 0.773 & 0.176 \\
\hline Negative & 251 & & \\
\hline Positive & 69 & & \\
\hline No. of positive LN & & 0.013 & 0.727 \\
\hline 0 & 224 & & \\
\hline 1 & 51 & & \\
\hline 2 & 26 & & \\
\hline$\geq 3$ & 19 & & \\
\hline EIC & & 0.760 & 0.544 \\
\hline Yes & 51 & & \\
\hline No & 246 & & \\
\hline Close RM & & 0.003 & 0.014 \\
\hline Yes & 13 & & \\
\hline No & 307 & & \\
\hline Molecular subtype & & 0.633 & 0.384 \\
\hline Luminal A & 211 & & \\
\hline Luminal B & 24 & & \\
\hline Triple negative & 18 & & \\
\hline HER2 & 66 & & \\
\hline Regional LN irradiation & & 0.854 & 0.603 \\
\hline Yes & 26 & & \\
\hline No & 294 & & \\
\hline Adjuvant systemic treatment & & 0.388 & 0.989 \\
\hline Yes & 300 & & \\
\hline No & 20 & & \\
\hline
\end{tabular}

LN, lymph node; EIC, extensive intraductal component; RM, resection margin; HER2, human epidermal growth factor receptor 2.

\section{Discussion and Conclusion}

ALN status is an important prognostic factor of survival outcome and a predictor of regional and systemic recurrence in breast cancer. Therefore, complete ALND is routinely performed in breast cancer patients regardless of early or advanced disease. Generally, eight or more axillary nodes should be routinely dissected irrespective of lymph node metastasis because many surgeons consider the number of dissected ALNs to be associated with accurate evaluation of ALN status. Despite the high locoregional and systemic control rates after ALND completion, most patients experience several complications, such as lymphedema, shoulder pain, and numbness. To reduce these complications, SNB was demonstrated by Giuliano et al. [11] to be an effective and minimally invasive alternative to ALND for identifying lymph node status. Thereafter, many studies investigated the efficacy and clinical outcomes of SNB, and SNB is now widely used in early breast cancer treatment.

In early breast cancer patients with negative SLN, no further ALND is recommended. In the case of sentinel node metastasis, most clinical practice guidelines recommend complete ALND. Although additional ALN involvement was found in 35\%-60\% of positive SLN patients, the axillary recurrence rate is reported to be $0.2 \%-0.9 \%$ for micrometastatic disease of SLN and around $1 \%$ for macrometastatic disease $[9,12-15]$. Based on these results, the hypothesis that ALN metastatic disease does not cause regional recurrence has been accepted. Therefore, several randomized phase 3 trials have been performed to investigate whether ALND can safely be omitted in early breast cancer patients with positive SLN. In the American College of Surgeons Oncology Group Z0011 trial, clinically node-negative early breast cancer patients with positive SLN were randomized to ALND or no ALND groups. At the median follow-up of 6.3 years, no difference was observed between groups for OS, progression-free survival (PFS) or locoregional PFS. The International Breast Cancer Study Group trial 23-01 trial was designed to determine whether no ALND was inferior to ALND in patients with one or more micrometastatic SLN with a tumor of maximum $5 \mathrm{~cm}$. After a median follow-up of 5 years, no difference was found between the ALND and no ALND groups for OS or PFS. In both trials, axillary recurrence in the no ALND group was about 1\%. In the AMAROS trial, ALND and axillary radiotherapy were compared. After a follow-up of 5 years, axillary radiotherapy was equivalent to ALND in OS, PFS, and axillary recurrence $[7,9,16]$. 
In the present study, our institutional practice achieved a high locoregional control rate through SNB and ALND. The accuracy and FNR of SNB are comparable to previously published studies $[17,18]$. Currently, ALND is not routinely performed in patients with negative SLN. Many surgeons insist that ALND should be carried out routinely in positive SLN patients because additional ALN involvement has been found in 35\%-60\% of patients. But axillary recurrence rate in patients with positive SLN was lower than expected in previous studies, even if patient underwent breast-conserving surgery without ALND.

In our institution, SNB was performed with high accuracy and low FNR. Sentinel node status was not associated with axillary recurrence. High locoregional control was achieved with SNB and ALND. Based on these results, axillary dissection might be individualized in early breast cancer patients according to sentinel node metastasis.

\section{Conflict of Interest}

No potential conflict of interest relevant to this article was reported.

\section{References}

1. Veronesi U, Paganelli G, Galimberti V, et al. Sentinel-node biopsy to avoid axillary dissection in breast cancer with clinically negative lymph-nodes. Lancet 1997;349:1864-7.

2. Krag DN, Anderson SJ, Julian TB, et al. Sentinel-lymphnode resection compared with conventional axillary-lymphnode dissection in clinically node-negative patients with breast cancer: overall survival findings from the NSABP B-32 randomised phase 3 trial. Lancet Oncol 2010;11:927-33.

3. Veronesi U, Viale G, Paganelli $G$, et al. Sentinel lymph node biopsy in breast cancer: ten-year results of a randomized controlled study. Ann Surg 2010;251:595-600.

4. Mansel RE, Fallowfield L, Kissin M, et al. Randomized multicenter trial of sentinel node biopsy versus standard axillary treatment in operable breast cancer: the ALMANAC Trial. J Natl Cancer Inst 2006;98:599-609.

5. Straver ME, Meijnen $P$, van Tienhoven $G$, et al. Role of axillary clearance after a tumor-positive sentinel node in the administration of adjuvant therapy in early breast cancer. $J$ Clin Oncol 2010;28:731-7.

6. Grube BJ, Giuliano AE. Observation of the breast cancer patient with a tumor-positive sentinel node: implications of the ACOSOG Z0011 trial. Semin Surg Oncol 2001;20:230-7.

7. Galimberti V, Cole BF, Zurrida $S$, et al. Axillary dissection versus no axillary dissection in patients with sentinel-node micrometastases (IBCSG 23-01): a phase 3 randomised controlled trial. Lancet Oncol 2013;14:297-305.

8. Giuliano AE, Hunt KK, Ballman KV, et al. Axillary dissection vs no axillary dissection in women with invasive breast cancer and sentinel node metastasis: a randomized clinical trial. JAMA 2011;305:569-75.

9. Giuliano AE, McCall L, Beitsch $P$, et al. Locoregional recurrence after sentinel lymph node dissection with or without axillary dissection in patients with sentinel lymph node metastases: the American College of Surgeons Oncology Group Z0011 randomized trial. Ann Surg 2010;252:426-33.

10. Donker M, Straver ME, van Tienhoven $G$, et al. Comparison of the sentinel node procedure between patients with multifocal and unifocal breast cancer in the EORTC 10981-22023 AMAROS Trial: identification rate and nodal outcome. Eur J Cancer 2013;49:2093-100.

11. Giuliano AE, Kirgan DM, Guenther JM, Morton DL. Lymphatic mapping and sentinel lymphadenectomy for breast cancer. Ann Surg 1994;220:391-401.

12. Veronesi U, Galimberti V, Paganelli G, et al. Axillary metastases in breast cancer patients with negative sentinel nodes: a follow-up of 3548 cases. Eur J Cancer 2009;45:1381-8.

13. Bilimoria KY, Bentrem DJ, Hansen NM, et al. Comparison of sentinel lymph node biopsy alone and completion axillary lymph node dissection for node-positive breast cancer. J Clin Oncol 2009;27:2946-53.

14. Naik AM, Fey J, Gemignani $M$, et al. The risk of axillary relapse after sentinel lymph node biopsy for breast cancer is comparable with that of axillary lymph node dissection: a follow-up study of 4008 procedures. Ann Surg 2004;240:46271.

15. van der Ploeg IM, Nieweg $O E$, van Rijk MC, Valdes Olmos RA, Kroon BB. Axillary recurrence after a tumour-negative sentinel node biopsy in breast cancer patients: a systematic review and meta-analysis of the literature. Eur J Surg Oncol 2008;34:1277-84.

16. Goodwin P. For patients with sentinel node-positive early breast cancer, RT as effective as and less toxic than complete surgical axillary clearance. Oncol Times 2013;35:33-4.

17. Miltenburg DM, Miller C, Karamlou TB, Brunicardi FC. Metaanalysis of sentinel lymph node biopsy in breast cancer. J Surg Res 1999;84:138-42.

18. Ban EJ, Lee JS, Koo JS, Park S, Kim SI, Park BW. How many sentinel lymph nodes are enough for accurate axillary staging in T1-2 breast cancer? J Breast Cancer 2011;14:296-300 